\title{
SOME MODEL CREATION RELATED QUESTIONS OF ENGINEERING
}

\author{
BÉla Csizmadia \\ Institute of Mechanics and Machinery, Szent István University \\ Páter K. u. 1., H-2103 Gödöllö, Hungary \\ Csizmadia.Bela@gek.szie.hu
}

[Received: September 29, 2015. Accepted: April 20, 2016]

Dedicated to Professor Barna Szabó on the occasion of his eightieth birthday

and to Professor Imre Kozák on the occasion of his eighty-fifth birthday

\begin{abstract}
Model creation is the most important challenge for an engineer. Consciously or intuitively, the engineer always creates models in the course of the creative process. However this subject is not discussed - or only marginally -, in the classroom or in the literature. The aim of this article is to contribute to the more conscious model-creating work. For the sake of this, this paper formulates some underlying principles. These principles must be observed during the model-creating work, and by presenting them during the university studies we can help the students to learn this important engineering task. Three of these principles are universal statements, relating to the recognition of the world, and two of them must be considered during the creation of a correct model. Three conclusions from the underlying principles are also conducted; these are important for the technical practice. The methods of mechanical model creation are shortly discussed at the end.
\end{abstract}

Keywords: Model creation, underlying principles

\section{TASK OF THE ENGINEER AND MODEL CREATION}

The engineer summarizes the knowledge of predecessors in order to recognize the problems of present days and to solve them. All these things are done for the purpose of building a more human future. Thus the engineer analyzes and describes both natural and human-made reality. The only way to carry out this task is by creating models, examining and comparing their results with reality.

Ultimately, the highest task of an engineer is to properly choose models. Nowadays, tons of calculating methods are available; although it does make a great difference what kind of model we choose to apply, since we can only apply them on models.

The model is a simplified form of reality, which, regarding the observed phenomenon and the determined goal, behaves similarly to reality in some features.

So a model must be created. This can be either carried out only into an intuitive way combined with great experience. Unfortunately this 'great experience' cannot be 
taught, but still it is highly important to put this knowledge in an integrated form and share it. At the same time the education of model creation appears barely or not at all in the engineering studies. So to say, it is quite difficult to teach as well. The author of this paper made an attempt to fill this need. To this end the author systematized the model creation method, and tried to draw up in a teachable, learnable form. The results of this attempt are summarized in this article.

Model creation appears in two ways in practice:

(a) "paid-off way", normal way, by the use of approximations;

(b) by a lot of experience combined with intuition.

ad a. As in the traditional - manual - calculation, also in the computation calculations (FEM) there are common models, such as the truss beams, which are modeled by links, or the design of bolts according to the shear stresses or facial pressure. In these cases - in a lower or higher level - the engineer carries out a general routine in the process of solving the problem. The applied theories and methods are mentioned in the subject of mechanics, machine elements and in other technical sciences during their studies. In the wide range of application of FEM the user investigates the solution or output and not the question of the precise approximation or the proper choice of the boundary conditions. The 'that's the way we do it' way of thinking overtakes the user, and one will not be aware of the right time to use it differently.

ad b. The other wide field of the engineering model creation is when the problem is not a routine task but something new. Although too many times the "paid off way" is used, unfortunately in a wrong way. Professor Barna Szabo made a survey in the United States about the efficiency of the use of finite element programs in the industry. According to the results of this survey, approximately half of the results evaluated by finite element method were wrong. And the error was not related to the finite element program itself, the source of the error was the wrong model: it did not described the reality. This is why we need to pay attention to the model creation problem.

The aim of this paper is not to deal with modeling of a concrete problem. I would like to summarize and to give methods and general principles for active technical intellectuals in order to apply and support the most important engineering process: model creation, based on my experiences acquired during my educational and research activities.

\section{General principles of model Creation, a possible description of the PHENOMENA}

Randomness and order govern the universe with the same weight and emphasis. This duality is the fuel of experimental science. The lack of randomness would cause the common logic to deterministically describe the universe (and the closer - broader reality) in its complexity without the need of observation. On the other hand, if this reality did not include some kind of order and logic, then science would have never come into existence. Let us take an example to understand this duality more deeply. 
The motions of gas particles in a closed vessel are entirely random, but the relation between its pressure, volume and temperature can be unequivocally determined. Randomness does not mean that there is no correlation between the motions of particles, but the initial conditions are random.

According to the unbreakable chain of randomness and order in the universe, in natural sciences - especially in engineering sciences - experimental and theoretical methods are mutually applied in research. This fact determines our knowledge about the physical world, because we can learn about the world based on two, well separated ways. One of them is direct experience, the other is the rational reasoning. Based on this, we can word the following:

1st Principle: One investigation must never be absolute according to the function of theory or experiments. Applying both at the same time will result in an adequate solution.

Nature is often said to be equal, united, and the world in it appears as a complete system, where everything is connected together. In a certain view this is true, but experiments show that even separated parts of reality can be precisely described without the knowledge of its entirety. What is the cause that gives us adequate information about the properly chosen parts? The reason is based on two peculiarity of the world: linearity and locality. A linear system - as is well known - can be easily investigated and described by two easily applied mathematical tools, addition and multiplication. However, using linear systems to determine a phenomenon is not obligatory in order to make the problem simpler. It is advised for use because it is possible. Naturally none of the systems are exactly linear. That fact raises a question: How is it possible to handle so many phenomena as a linear system? There are three reasons:

- In many cases the non-linear effects are very weak,

- The range of influence of non-linear effects is strongly determined,

- Many linear systems are not sensitive to weak perturbances.

Linearity exists in most cases, but it must be identified among the parameters.

The other peculiarity that makes the examination of the properly identified parts possible without the knowledge of the whole, is the locality. This means that in most cases the behavior of the physical systems is entirely influenced by the effects (external forces, moments, etc) of their very surroundings.

2nd Principle: Reality can be dissembled and information can be gained by only examining separated parts and not the whole.

According to these observations and the 2nd principle, the following conclusion can be drawn:

In model creation, a very important step is to make clear what we wish to measure in what function.

As we mentioned above, there are two, well-separated ways to gain information about the world. One is direct experience; the other is rational reasoning. The fall 
of an apple can be an example. Sensory perception, which is a direct experience, can provide measured data about the fall of an apple. It is also possible to gain more information about this phenomenon, in a different and deeper way. By the combination of Newton's laws and some mathematical skills a model can be created to investigate the falling apple. This model is not based on our sensory perception but on our mind which - by the use of the earlier universalized experiences and laws - finds the correlation between the once fallen apple and the deeper, comprehensive physical processes. Now the examination of the mechanical model is possible with the applied physical-mathematical model. Although it is unseen, still - in its abstract way - it is able to provide information about reality in a higher, more general way. At the same time these laws were and are created by people based on their sensory perceptions. The 3rd principle is applied to these natural laws. Here it must be highly emphasized that natural laws were not made by mankind but discovered. The laws are independent, and they always existed.

3rd Principle: Natural principles are absolute and irrespective of space, entirely valid in time, and it is possible to involve them in a mathematical form. They are independent of the observer, and the condition of the phenomena. The parts of nature are not abstract mathematical formations.

Thus if we engineers create a model by observing a phenomenon and we lay down new formulas, these are not natural laws. They do not operate in every case. This conclusion provides a new principle to engineer researchers:

According to the given results and the model, the limits of applicability of the model must be determined.

\section{BASIC PRINCIPLES OF MODEL CREATION}

Creating a model raises one question: abstraction. Abstraction is not solely an engineering activity or method but a fundamental skill of humanity. Let us explain it with an example. If a father tells his child - who probably does not even talk - to sit down on a chair in a foreign environment, the child will most likely go to a chair and sit on it. Not on a pillow, ground or a couch, but on a chair. He will sit on a chair even if he has never seen that chair before, irrespectively of whether the chair has three or four legs, with or without a back, upholstered or not, smooth, fret worked, wooden or iron, etc.

Basically he recognizes the chair as an abstracted concept, and he does not look for a certain object. Abstraction is also the fundamental existence of mathematics. In order to say 'five stones and two stones are seven stones' we have to neglect the form, material structure and color. The concept of stone must be abstracted, where the stones are isolated substances.

Obviously the concept of the examined or observed object or phenomenon must be determined by taking into consideration the observing purpose. Let us investigate this statement with another simple example. If we wish to analyze a chair, furniture or other wooden structure, different denotations must be addressed to each of them. 
All of them have different models. Some peculiarities are highlighted and concerned dominant and others are neglected according to the purpose of modeling.

When we examine a phenomenon, not only the phenomenon itself, but the aim of our investigation is also important. The limits of applicability must also be taken into account based on this.

4th Principle: The model is also determined by the aim of investigation.

Ultimately let us talk in general about the relation of model and reality. As we have already declared, the model is a simplified form of reality which behaves similarly to reality in some features. Similarly, but not entirely exactly! The difference is the error of the model creation. What is the origin of this error or difference between the model and reality? Firstly, the model describes the order of reality and neglects its randomness. Secondly, the model only takes into account order determining parameters respective to the chosen observing aims, so we are not talking about an entire analogy. Thirdly, there can be differences in the calculations of the created model, since there are approximations in the equations and so also in the solutions.

Ultimately the result is not the same between reality and the model. The cause of the difference must be found in the model, not in the natural laws. Let us verify it with an old, astromechanic example. The law of mass attraction is well known. Taking into account the Sun and the planets of our solar system, a model was created about the motion track of Neptune. According to the model - taking into account the law of mass attraction - the calculated result did not correspond with the astronomers' observation. Maybe the law of mass attraction did not work there? Or some important feature was neglected in the model? Eventually, they came to the following conclusion: one external effect caused the anomaly in the motion. Then they appointed a certain position at a certain time in the sky looking for the mysterious perturbance. Thus they discovered Pluto. Therefore the model was incorrect for determining the motion track of Neptune. That is why the calculated result did not correspond with reality.

5th Principle: The created models provide new information about the world, but these results must be always compared with reality and the chosen aim. Decisions about the adequateness of the model must be based on the collective results.

According to the 4th and 5th principles a conclusion can be drawn for the technical practice:

the correct model is as simple as it can be, but properly approximates reality regarding the aims for examinating.

\section{Methods of MechaniCAL MOdel CREATION}

We have already discussed the concept that the phenomena and the examining aims determine the model, and also that the model is as simple as possible. The purpose of model creation has been also investigated. Let us discuss what methods are possible to use in order to create models. The following investigations - regarding the examples - will be limited to mechanical models. Three methods are adequate to reach our goals. 
4.1. Analytical 'exact' models. The 'exact' word in the subhead means that the error of the model is within the range of the limited margin of error regarding the aim of examination. In these models the estimation or error is generally based on hundreds of years engineering experience. The complete model can be obtained by these experience verified modelparts. Intuition and the engineering experience have a great relevancy in these cases. Most of the routine engineering models are analytical.

One great feature of the analytical models is the relatively easy parameter identification. Parameter identification reveals what effects the gradient of the parameters perform on the phenomena. This method easily allocates the dominant parameters in a given domain. In addition the parameter identification allows the optimum search algorithm, which can be essential in economical or feasibility aspects.

4.2. 'Approximating' models. Modeling extremely complex phenomena adduces the application of an initial, but not entirely accurate - regarding the examining aims - model. Most of the times these models are not created by necessity, but after mature deliberation. Let us investigate the reasons that may cause the application of approximating models!

Concept of successive approximation. The engineer does not always find an adequate model. Thus one must start with the model which seems to be the simplest, and by examining the result, a conclusion must be drawn whether it is necessary to use a more advanced model. Step by step, the engineer approaches the simplest, usable and precise enough model. It is also possible if one does not find out all the approximating models, but creates only the proper model.

Complex solution. One problem can be approached in a complex way as well: this is the collective application of theoretical and experimental models. This method is often used in research when the solution of the problem requires experimental methods. Not even in these cases should we surrender the numerical, mechanical, theoretical model creation, even if the model is a coarse approximation of reality. The possible (sometimes really coarse, or just not accurate enough) approximating model must be created since it can greatly support the description of the phenomena in two ways:

- to create the possibility of the equation analysis and the application of analogy laws, or to draw up the model laws which are helpful in the evaluation of the experimental results.

- to decrease the number of parameters and allow the approximation with productfunction:

$$
y=f_{1}\left(x_{11}, x_{12}, \ldots\right) f_{2}\left(x_{21}, x_{22}, \ldots\right)
$$

where the function $f_{2}$ is the solution of the approximating model, while

$$
y\left(x_{11}, \ldots, x_{21}\right)
$$

is the experimental result and $f_{1}\left(x_{11}, x_{12}, \ldots\right)$ is the result of the experiment, which can be used for the determination of the coefficient $f_{1}$. 
Let's see a simple, elementary example to shed light on this, of course, this example is not a task to be implemented. Suppose that we have a cantilever beam fixed at one of its ends by screws. We would like to evaluate the deflection of the beam's endpoint, because we do not know the rigidity of its support. However, we do not want to determine the concrete deflection of one given beam, but the behavior of the whole system. We have a computational model for the determination of the deflection:

$$
y=\frac{f \ell^{3}}{3 I E}=\frac{1}{3} f_{2}\left(x_{21}, x_{22}, \ldots\right)
$$

This equation is not a model for the problem, because the number, the size, position and the screwing torque of the screws and the rigidity of the support can modify the deflection. Suppose that we would like to describe these effects by measurements, i.e. we would like to introduce a changing variable instead of the $1 / 3$ multiplier. This changing variable will be determined as a function of measurements:

$$
y=f_{1}\left(x_{11}, x_{12}, \ldots\right) \frac{f \ell^{3}}{I E}
$$

Based on this method, we get a general, complex solution. Of course, this method is not used for such simple problems, this is just an illustrative example.

Model creation for experimental model. In this case not the mechanical but the experimental is the approximating model. If we wish to verify the behavior of an existing mechanical structure - taking into consideration the specifications - then the experimental investigations may turn out to be extremely costly. Thus we carry out the investigations on - a fairly proper - simpler experimental model. Even in this case a theoretical model must be created for the description of the approximating experimental model in order to verify the applicability of the experimental model.

Parameter identification. This is the most peculiar and important case of the approximating models. The examining aim is so complex that it is impossible to describe it with an analytical model. Here the possible use of a numerical model makes an appearance regarding the given mechanical structure. At the same time if the aim is not verification but design, then first a construction must be achieved. This is often determined by earlier experience of the designer. Rationally first an approximating analytically solvable model must be set up in order to carry out parameter identification. After the identification, optimization must be undertaken. The construction designed in this manner now is ready to be investigated with the more precise numerical model. If the results need more modification then it will take far less effort. This method is quite efficient and successful.

4.3. Numerical models. In the previous subhead the possibility to approximate reality with numerical models has been already declared. This numerical model can be either the result of a former approximating model, or a directly created model as well. Primarily the latter is used in verification, in those cases when not the mechanical model is dominant in the determination of the construction.

So far, only a few numerical models are suitable for optimization (one example is the evolutional model), and their capabilities are also limited to a narrow range 
of application. These problems cannot be solved other way. At this moment these models represent the most complex methods of model creation.

Finally we have to draw attention to the fact that numerical models are not suitable to solve complex engineering problems in general. They can act as supplement in the application and they complements the possible applicable tools, but the given results must be understood, defined, and put into context in the whole by the engineer. The problem cannot be solved either by computer, or with excellent program systems, but only by creative engineer.

\section{EduCATional application of the MOdel CREATION'S BASiC PRINCIPLES}

The author of this article summarized the basic principles in a book titled Model Creation. This book is the fourth in the textbook series Mechanics for Engineers. After the detailed exposition of the basic principles and presentation of basic examples, the book - written by several authors - presents a large number of case studies. These case studies are the solutions of real industrial problems, and there are references within them to the basic principles of model creation, and their implementation. The textbook is for the compulsory subject called Model creation in the MSc engineering program. After studying the basic principles, all the students had the task of analyzing a structure and the creation of all of its models. For solving this, the students had to use all of their mechanical knowledge studied in the previous five semesters and the information written in the textbook. Thus, they had an integrated, creative task that could only be solved by participating in large number of personal consultations. It is my experience and belief, that in this way the students gained knowledge on the creative engineering tasks, and the earliest possible learning of more effective engineering work. 